\title{
Fractionation of organic matters in sludge rejected by NF membrane
}

\author{
B.N. Tsai ${ }^{\mathrm{a}}$, C.H. Chang ${ }^{\mathrm{a}}$, D.J. Lee ${ }^{\mathrm{a} *}$, J.Y. Lai $^{\mathrm{b}}$ \\ ${ }^{a}$ Department of Chemical Engineering, National Taiwan University, Taipei 10617, Taiwan \\ Tel. +886(2)23625632; Fax +886(2)23623040; email: djlee@ntu.edu.tw \\ ${ }^{b}$ R\&D Center for Membrane Technology and Department of Chemical Engineering, \\ Chung Yuan University, Chungli 320, Taiwan
}

Received 1 August 2007; accepted revised 25 September 2007

\begin{abstract}
Nanofiltration membrane bioreactor (NF-MBR) was proposed to provide quality effluent free of virus and most large organic substances. The dissolved organic matter (DOM) in soluble microbial products (SMP), loosely bound extracellular polymeric substances (LBEPS) and tightly bound extracellular polymeric substances (TBEPS) of wastewater sludge before and filtered through nanofiltration membrane were fractionated using size exclusion chromatography, characterized with ultraviolet absorbance at 230, 254 and $280 \mathrm{~nm}$, dissolved organic carbon (DOC) content, and excitation-emission-matrix (EEM) spectra. Except for long-chain organic acids, the membrane rejected most organic matters in SMP and LBEPS fractions. However, the membrane failed to reject fluorescent DOM from TBEPS at molecular weight of 3000-7000 Da. The content of TBEPS in the mixed liquor affected significantly the effluent quality in NF-MBR application.
\end{abstract}

Keywords: EPS; SMP; Sludge; SEC; EEM; DOC; Filtration

\section{Introduction}

Activated sludge liquor is composed primarily of living cells, extracellular polymeric substances (EPS), inert biomass and soluble microbial products (SMP) [1]. Interactions between SMP, biomass and inert biomass were elucidated by

*Corresponding author.
[2-4]. The EPS are differentiated into extractable EPS, the EPS fraction bound tightly to solid surfaces, and soluble EPS (also called slime polymers), the fraction characterized by free movement between sludge flocs and surrounding liquor [5]. Other classification paradigms classify EPS as "loosely bound" (LB) and "tightly bound" (TB) fractions [6]. Li and Yang [7] recently reported that quantity of loosely bound

Presented at the Fourth Conference of Aseanian Membrane Society (AMS 4), 16-18 August 2007, Taipei, Taiwan. 
EPS correlated with flocculation and sedimentation in activated sludge. Laspidou and Rittmann (2002) proposed that the soluble EPS from sludge was equivalent to its SMP fraction. Ramesh et al. $[8,9]$ extracted SMP and soluble EPS from wastewater sludge and compared the physicochemical characteristics of the SMP and soluble EPS from original and aerobically or anaerobically digested wastewater sludge. These authors noted that reversible and irreversible fouling of membrane filtration of wastewater sludge was mainly attributable to the LBEPS fraction of the sludge. In the above studies, extracted SMP and EPS fractions were tested before detailed fractionation of the dissolved organic matter (DOM).

Most membrane bioreactor (MBR) employs microfiltration (MF) or ultrafiltration (UF) membranes. New generation of MBR is proposed with the use of nanofiltration membrane in order to remove completely virus and most large organic substances from effluents. The rejection characteristics by the nanofiltration (NF) membranes are dependent on molecular size as well as surface charge of the target solutes, since the membrane itself often carries charge at different $\mathrm{pH}$ conditions [10,11]. Ref. [12] explained the inorganic rejection behavior by the charge effect on nanofiltration membranes. Schafer et al. [13] also explained the rejection characteristics of organic matter and salts are depending on membrane charge effect and other parameters. Ref. [14] studied the rejection of limited number of endocrine disrupting compounds (EDCs) and pharmaceutical active compounds (PhACs) by polyamide NF/RO membranes. Several workers [15-17] also discussed the relationships between the physical/chemical properties of compounds and their rejection by NF/RO membranes. Thanuttamavong et al. [18] described the nanofiltration mechanism of organic matter by size exclusion, while electrostatic repulsion plays important role for inorganic rejection. Liikananen et al. [19] reported that, in NF the rejection of organics not only depends on the sieving effect, but also on the charge and hydrophobicity effects between the membrane and organic compound. Ref. [20] reported that the rejection of organic matters by RO and NF membranes depends on the pore size distribution and effective number of pores in the membrane. Ref. [21] noted significant correlation between molecular weight cut off (MWCO) and the rejection of natural organic matters. Several workers [22-24] reported that fouling after pore blockage (or constriction) could enhance the organic rejection. Humic acids at MW of $30-50 \mathrm{KD}$ were minimally rejected by clean membrane [23]. Concentration polarization increases osmotic pressure, hence reducing transmembrane pressure, decrease the rejection rates of total dissolved solids, and biochemical and chemical oxygen demands [25]. Schaep et al. [26] reported that nanofiltration is very effective process for removal of hardness. The removal of fluoride and aluminum were efficient at applied pressure of 12 bars using NF membrane [27].

Size-exclusion chromatography (SEC) employs liquid chromatography to measure molar-mass distribution of natural and synthetic macromolecules. The analytes with the highest molar masses are eluted from the separation column before the smaller molecules, which are retained longer within the column. In this study, the DOM in the extracted SMP and LB- and TBEPS from wastewater sludge and those in permeate from an NF module were fractionated using SEC. The fractionated DOM were characterized by ultraviolet absorbance, dissolved organic carbon (DOC) content and excitation-emission-matrix (EEM).

\section{Experimental}

\subsection{Sample and filtration test}

The test sample was collected from the return sludge stream of the wastewater treatment system at Neili Bread Plant, Presidential Enterprise Co. Taoyuan, Taiwan. The chemical oxygen demand (COD) and suspended solids (SS) data for the sludges, measured via EPA Standard Methods, 
were 16,000 and $10,000 \mathrm{mg} \mathrm{L}^{-1}$, respectively. The supernatant drawn from settled sludge had a $6.7 \mathrm{pH}$ and $86.7 \mathrm{mg} \mathrm{L}^{-1}$ soluble COD. Fine particles in the supernatant exhibited a zeta potential of $-24.7 \mathrm{mV}$. Chemical analysis of the sample revealed $\mathrm{C}: \mathrm{H}: \mathrm{N}: \mathrm{O}=41.3: 6.6: 5.4: 46.7$. Fat, protein and carbohydrate content were $0.04 \mathrm{~g}, 0.43 \mathrm{~g}$, and $0.56 \mathrm{~g}$, respectively, per gram of dried solids.

The supernatant of each of sludge sample was first separated from solid phase by centrifugation at $6000 \mathrm{~g}$ for $10 \mathrm{~min}$. The resulting supernatant was combined with two volumes of acetone and maintained at $4{ }^{\circ} \mathrm{C}$ for $24 \mathrm{~h}$ to precipitate soluble substances. The collected precipitate was designated the sludge SMP for experimental purposes.

After centrifugation, the resulting dewatered cake was re-suspended in a $0.85 \% \mathrm{w} / \mathrm{w} \mathrm{NaCl}$ solution with several glass beads then sonicated at $20 \mathrm{kHz}$ and $330 \mathrm{WL}^{-1}$ for $2 \mathrm{~min}$, shaken horizontally at $120 \mathrm{rpm}$ for $10 \mathrm{~min}$ then sonicated again at the same power for an additional $2 \mathrm{~min}$. The liquor was centrifuged at $8000 \mathrm{~g}$ for $10 \mathrm{~min}$ to separate solids and supernatant. The supernatant was combined with two volumes of acetone and maintained at $4{ }^{\circ} \mathrm{C}$ for $24 \mathrm{~h}$ to precipitate soluble substances. The collected precipitate was designated the loosely bound EPS (LBEPS) of the sludge sample.

The residual solids remaining after the above test were resuspended in a $0.85 \% \mathrm{NaCl}$ solution, sonicated for $2 \mathrm{~min}$ then heated at $80^{\circ} \mathrm{C}$ for $30 \mathrm{~min}$ to collect supernatant. The obtained supernatant was mixed with 2 vol of acetone and maintained at $4{ }^{\circ} \mathrm{C}$ for $24 \mathrm{~h}$. The collected precipitate was designated the tightly bound EPS (TBEPS) in the sludge sample. Preliminary tests revealed that $20 \mathrm{~min}$ of heat extraction was sufficient to obtain all extractable EPS (TBEPS).

A turbidimeter (HACH Model 2100 AN) measured turbidity in the water samples. The zetasizer (Zetasier 2000, Marlvern) measured zeta potentials of suspended particles. The $\mathrm{pH}$ of the samples was measured using a calibrated pH meter (JENCO 1671).
Dead-end filtration test was conducted using NF membrane made of poly ether sulfone of MWCO of 15,000 Da. The constant pressure filtration was conducted with fixed applied pressure of 9 bars.

\subsection{Size exclusion chromatography}

The HPLC-SEC fractionation system was comprised of a BETA 10 Gradient pump (Ecom spol. s r. o., Prague, Czech Republic), a size exclusion HW-50S column (TOYOPEARL resin with $20-40 \mu \mathrm{m}$ particle size, TOSOH Bioscience LLC, Montgomeryville, PA, USA), an on-line SAPPHIRE 600 UV-VIS variable wavelength detector (Ecom spol. s r. o., Prague, Czech Republic), an IOTA 2 refractive index detector (Precision Instruments, Marseille, France) and a CHF 100SA fraction collector (Advantec MFS, Inc., Dublin, CA, USA). The SEC effluent was collected at 3-min intervals and probed for fluorescence excitation and emission matrix (EEM) and dissolved organic carbon (DOC) content. The TOC Analyzer (Aurora Model 1030, OI Analytical, College Station, TX, USA) measured DOC at a detection limit of $0.002 \mathrm{mg}_{-} \mathrm{CL}^{-1}$. The flow rate of the pump for SEC test was $1 \mathrm{~mL} \mathrm{~min}^{-1}$. The column was operated with a phosphate mobile phase $\left(0.0024 \mathrm{M} \mathrm{NaH}_{2} \mathrm{PO}_{4}+0.0016 \mathrm{M}\right.$ $\mathrm{Na}_{2} \mathrm{HPO}_{4}, \mathrm{pH}$ 6.8) containing $0.025 \mathrm{M} \mathrm{Na}_{2} \mathrm{SO}_{4}$ at ionic strength $0.1 \mathrm{M}$. Polyethylene glycols (PEGs, 200, 1000, 4000, 8000 and 20,000 $\mathrm{g} \mathrm{mol}^{-1}$ ) were used for MW calibration of chromatograms. The absorbance wavelength of UV detector was set at $254 \mathrm{~nm}$. A $2 \mathrm{~mL}$ sample volume was injected into the column. The UV and RI data were collected every $0.05 \mathrm{~s}$. Recovery of SEC effluent after 150 min retention was $97-104 \%$. The fractions were designated A-H according to collected elution time. Specifically, fraction A has DOM of MW $>20,000 \mathrm{Da}$. Fractions B-H collected DOM of MW range 8000-20,000, 3500-8000, 2000-3500, 1200-2000, 500-1200, 200-500 and <200 Da, respectively. 


\subsection{Excitation-emission matrix}

The fluorescence EEM spectra of leachate samples were measured by a Cary Eclipse fluorescence spectrophotometer (Varian Inc., Palo Alto, CA, USA). The EEM spectra were collected with scanning emission wavelength of $250-600 \mathrm{~nm}$ at $2 \mathrm{~nm}$ increments and excitation wavelength of $200-500 \mathrm{~nm}$ at $10 \mathrm{~nm}$ increments. The spectra were recorded at a scanning rate of $1200 \mathrm{~nm} \mathrm{~min}{ }^{-1}$ using excitation and emission slit bandwidths of $5 \mathrm{~nm}$. Before each scan, the cuvettes were rinsed and ultrasonicated with $5 \%(\mathrm{w} / \mathrm{w})$ nitric acid solution. The washed liquor of the cuvettes, the Milli-Q water and the effluent of mobile phase from SEC were scanned before each fluorescence analysis to prevent

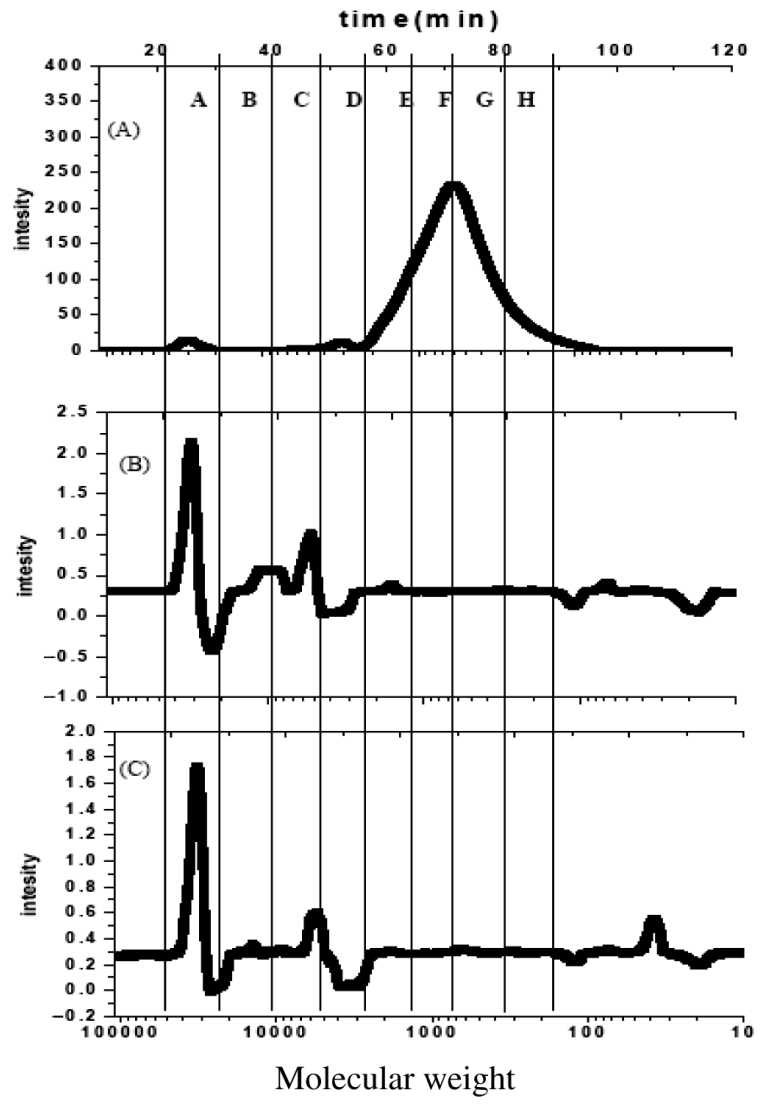

(a) contamination. In accordance with the method employed by Baker (2001), the EEM were analyzed at $25 \pm 2{ }^{\circ} \mathrm{C}$, and blank water scans were run every 5-15 analyses using Milli-Q water. The Raman peak of water at $348 \mathrm{~nm}$ was used to test machine stability and to permit interlaboratory comparison. The intensities yielded from Rayleigh and Raman scatters were subtracted from the collected scanned data.

\section{Results}

\subsection{Original sample}

Fig. 1 shows the MW distributions of DOM in the TBEPS sample according to fractional UV absorbance at 230, 254 and $280 \mathrm{~nm}$, respectively.

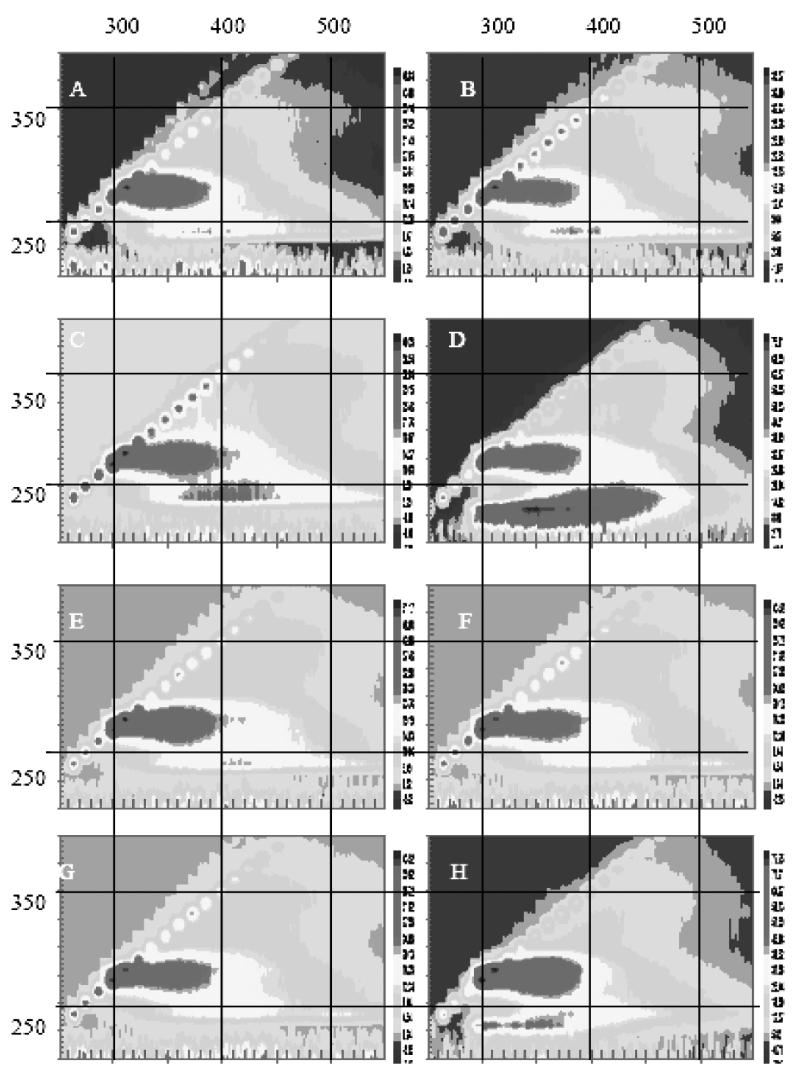

(b)

Fig. 1. SEC chromatograms (up) (A) $230 \mathrm{~nm}$, (B) $254 \mathrm{~nm}$, (C) $280 \mathrm{~nm}$ and EEM spectra (down) of TBEPS sample. 
There are two peaks (MW $>20,000$ and $2600 \mathrm{Da})$ at $230 \mathrm{~nm}$, four peaks $(>20,000,8000,4000$ and $1600 \mathrm{Da})$ at $254 \mathrm{~nm}$, and three peaks $(>20,000$, $4500,<200 \mathrm{Da})$ at $280 \mathrm{~nm}$ for the TBEPS sample. Fig. 2a lists the MW distribution based on fractional DOC values. The fraction $\mathrm{E}$ (1200-2000 Da) exhibited the highest DOC $(25.2 \%)$ of all fractions. The characteristics of SMP and LBEPS were not discussed herein.

Fig. 1 also shows the EEM spectra for fractions A-H of the TBEPS sample. Two high intensity bands, $\mathrm{Ex} / \mathrm{Em}=280 / 300-380 \mathrm{~nm}$ and

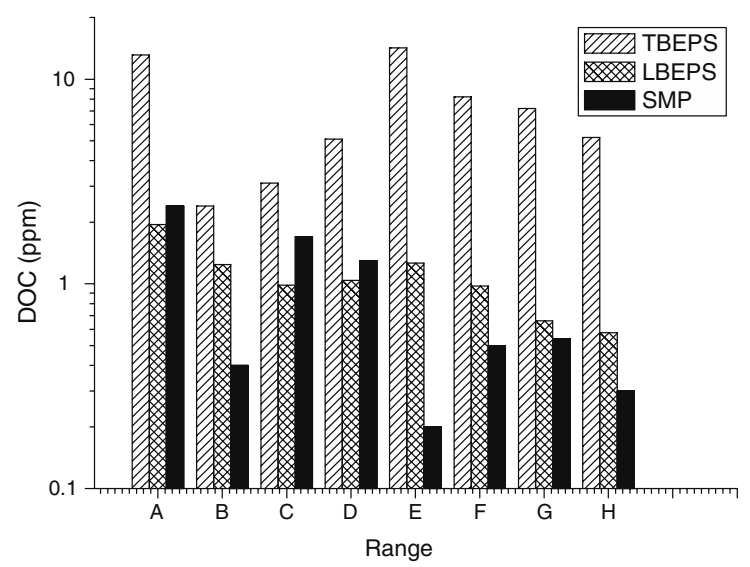

(a)

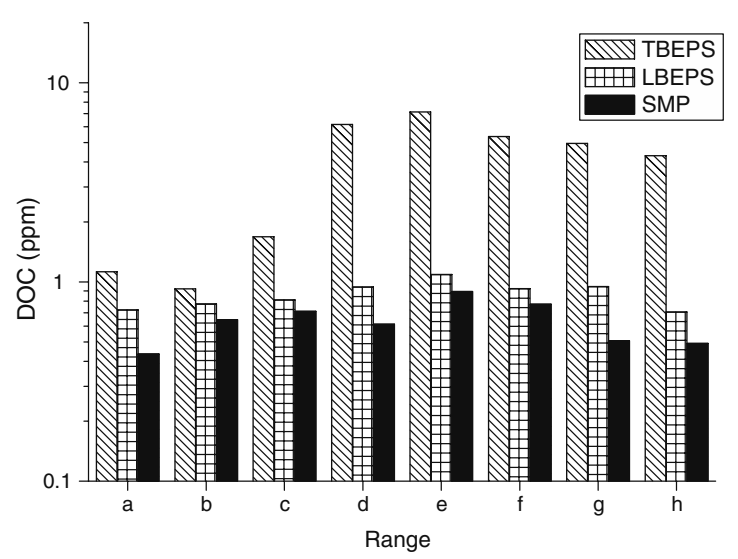

(b)

Fig. 2. The SEC-DOC distributions of SMP, LB and TBEPS of wastewater sludge. (a) Original sludge; (b) membrane filtration permeate.
220/300-380 nm, corresponded with regimes IV and I-II by Chen et al. [28], respectively. In some fractions, weak intensity regimes were noted for humic substances at 450/490 nm.

\subsection{Organic rejection via membrane}

Most SEC-UV peaks in the permeate following membrane filtration were removed using the SMP and LBEPS except for those at MW 200-2000 Da excited at UV $230 \mathrm{~nm}$ (data not shown). The SEC-DOC distributions (Fig. 2b) revealed that the DOC in all SEC fractions for SMP ranged $0.3-0.9 \mathrm{mg} \mathrm{L}^{-1}$. Hence, the membrane filtration rejected most DOM in both SMP and LBEPS. The remaining fluorescent compounds were long-chain organic acids. Restated, the current membrane could not effectively reject organic acids in the SMP and LBEPS fractions

The permeate for TBEPS test (Fig. 3) contained more DOM than those for SMP and for LBEPS (Fig. 2b). Except for the long-chain organic acids as detected in the SMP and LBEPS tests, the humic-acid like (UV254) and protein-like (UV280) substances at MW of 3000-7000 Da were noted in the TBEPS permeate. The EEM spectra shown in Fig. 3 also revealed the presence of proteins and humic substances. The SEC-DOC distributions (Fig. 2b) revealed high contents of organic carbon of 8-9 mg L $\mathrm{mg}^{-1}$ in the fractions D-F. Hence, the rejection of organic matters by NF membrane depends not only on the pore size, but also strongly on the corresponding biological origin. The advantages proposed by NF-MBR could be significantly challenged by the TBEPS fractions of the wastewater sludge.

\section{Conclusion}

This study fractionated the DOM into SMP, LBEPS and TBEPS fractions from a wastewater sludge using size exclusion chromatography, and filtered the extracted DOM using NF membrane. 


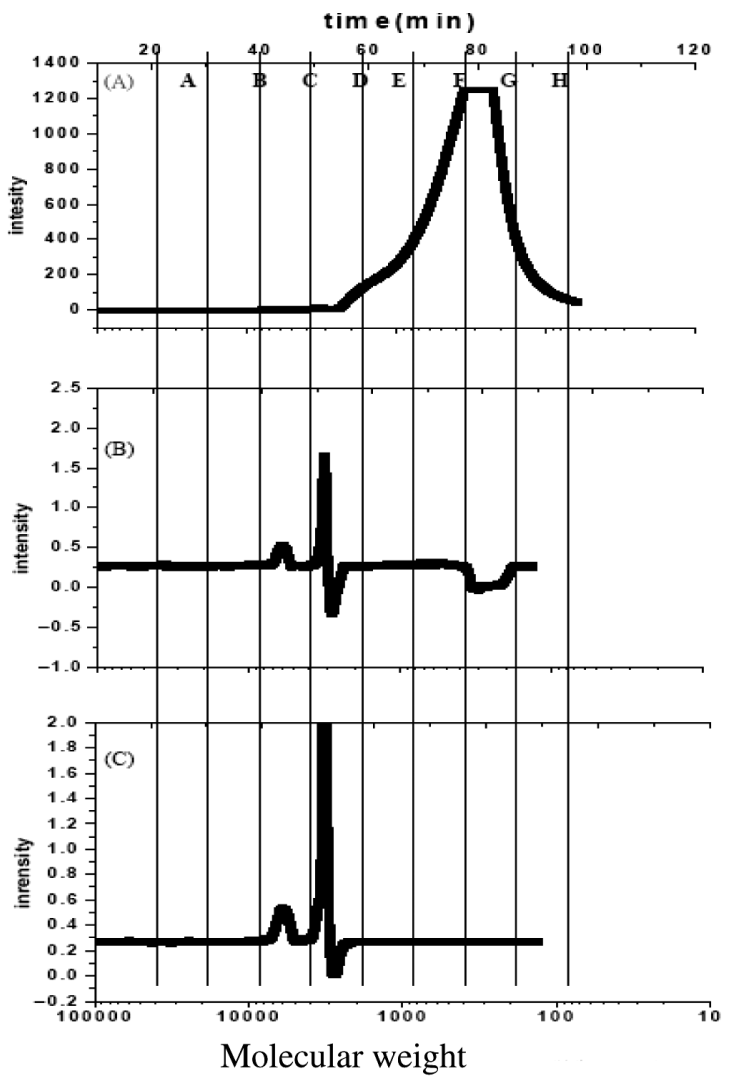

(a)

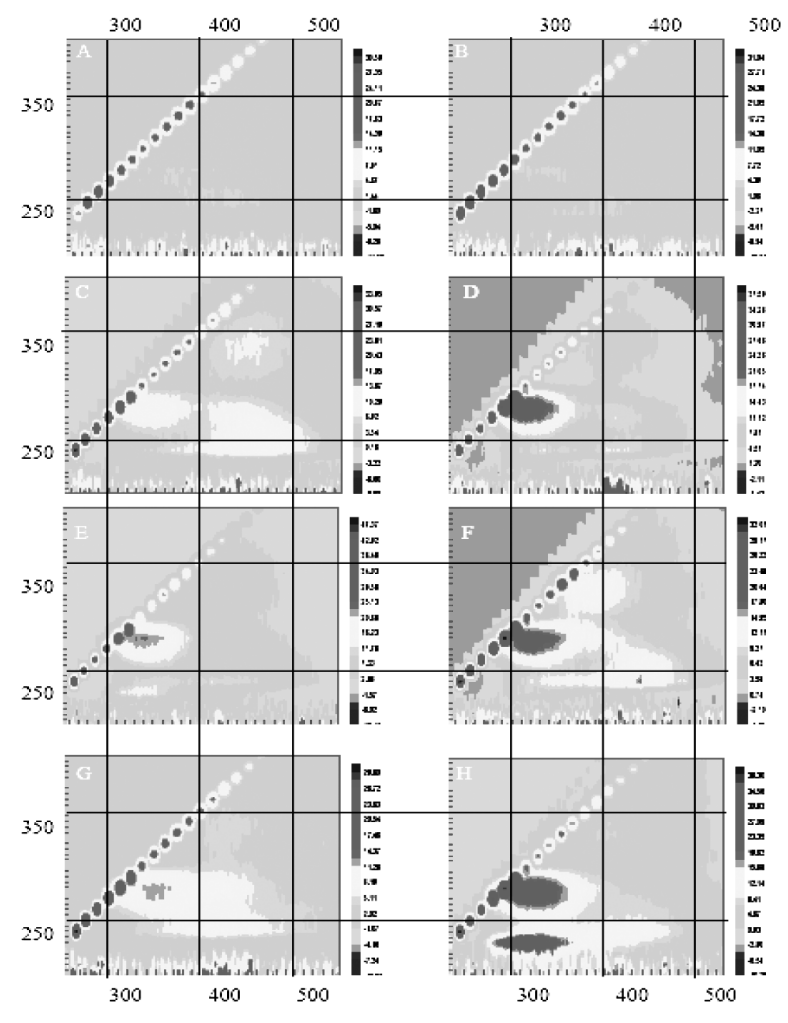

(b)

Fig. 3. The SEC chromatograms (LEFT) (A) $230 \mathrm{~nm}$, (B) $254 \mathrm{~nm}$, (C) $280 \mathrm{~nm}$ and EEM spectra (right) of TBEPS sample.

The DOM were characterized using ultraviolet absorbance at 230, 254 and $280 \mathrm{~nm}$, DOC content, and EEM spectra. Following NF filtration, most of UV fluorescent peaks in SMP and LBEPS fractions were removed, indicating that the filtration effectively rejected these fluorescent DOM in wastewater sludge. However, long-chain organic acids were not rejected as detected in the SEC-UV spectra. Moreover, the NF filtration failed to remove the organic matters in TBEPS at MW 3000-7000 Da and with strong fluorescent intensities at 254 and $280 \mathrm{~nm}$. Hence, the use of PES NF membrane in NF-MBR application may face trouble to deal with these organic matters in suspended liquor.

\section{References}

[1] C.S. Laspidou and B.E. Rittmann, A unified theory for extracellular polymeric substances, soluble microbial products, and active and inert biomass, Water Res., 36 (2002) 2711-2720.

[2] H. Furumai and B.E. Rittmann, Advanced modeling of mixed populations of heterotrophs and nitrifiers considering the formation and exchange of soluble microbial products, Water Sci. Tech., 26 (1992) 493-502.

[3] E. Namkung and B.E Rittmann, Effects of SMP on biofilm reactor performance, J. Environ. Eng., 114 (1988) 199-210.

[4] G.E. Speitel, K. Dovantzis and F.A. Digiano, Mathematical modeling of bioregeneration in GAC columns, J. Environ. Eng., 113 (1987) 32-48. 
[5] S. Rosenberger and M. Kraume, Filterability of activated sludge in membrane bioreactor, Desalination, 151 (2002) 195-200.

[6] T.L. Poxon and J.L. Darby, Extracellular polyanions in digested sludge: measurement and relationship to sludge dewaterability, Water Res., 31 (1997) 749-758.

[7] X.Y. Li and S.F. Yang, Influence of loosely bound extracellular polymeric substances (EPS) on the flocculation, sedimentation and dewaterability of activated sludge, Water Res., 41 (2007) 1022-1030.

[8] A. Ramesh, D.J. Lee and S.G. Hong, Soluble microbial products (SMP) and extracellular polymeric substances (EPS) from wastewater sludge, Appl. Microbiol. Biotech., 73 (2006) 219-225.

[9] A. Ramesh, D.J. Lee and J.Y. Lai, Membrane biofouling by extracellular polymeric substances or soluble microbial products from membrane bioreactor sludge, Appl. Microbiol. Biotech., 74 (2007) 699-707.

[10] K. Ikeda, H. Hachisuka, T. Nakamura, S. Kimura and K. Ueyama, Zeta-potential of fouled thin film composite membrane, J. Chem. Eng. Jpn., 32 (1999) 581-587.

[11] A.E. Childress and M. Elimelech, Relating nanofiltration membrane performance to membrane charge (electro kinetic) characteristics, Environ. Sci. Tech., 34 (2000) 3710-3716.

[12] C. Ratanatumskul, T. Urase and K. Yamamoto, Description of behavior in rejection of pollutants in ultra low-pressure nanofiltration, Water Sci. Tech., 38 (1998) 453-462.

[13] A.I. Schafer, A.G. Fane and T.D. Waite, Nanofiltration of organic matter: removal, fouling and influence of multivalent ions, Desalination, 118 (1998) 109-122.

[14] K. Kimura, G. Amy, J.E. Drewes, Th. Heberer, T.U. Kim and Y. Watanabbe, Rejection of organic micropollutants (disinfection by-products, endocrine disrupting compounds, and pharmaceutically active compounds) by NF/RO membranes, J. Memb. Sci., 227 (2003) 113-121.

[15] H. Ozaki and H. Li, Rejection of organic compounds by ultra-low pressure reverse osmosis membrane, Water Res., 36 (2002) 123-130.

[16] B. Van der Bruggen, D. Schaep, C. Wilms and C. Vandecasteele, Influence of molecular size, polarity and charge on the retention of organic molecules by nanofiltration, J. Memb. Sci., 156 (1999) 29-41.

[17] K. Kimura, S. Toshima, G. Amy and Y. Watanabe, Rejection of neutral endocrine disrupting compounds (EDCs) and pharmaceutical active compounds (PhACs) by RO membranes, J. Memb. Sci., 245 (2004) 71-78.

[18] M. Thanuttamavong, K. Yamamoto, J.I. Oh, K.H. Choo and S.J. Choi, Rejection characteristics of organic and inorganic pollutants by ultra lowpressure nanofiltration of surface water for drinking water treatment, Desalination, 145 (2002) 257-264.

[19] R. Liikanen, I. Miettinen and R. Laukkanen, Selection of NF membrane to improve quality of chemically treated surface water, Water Res., 37 (2003) 864-872.

[20] K. Koustic and B. Kunst, RO and NF membrane fouling and cleaning and pore size distribution variations, Desalination, 150 (2002) 113-120.

[21] M. Taniguchi, J.E. Kilduff and G. Belfort, Modes of natural organic matter fouling during ultrafiltration, Environ. Sci. Tech., 37 (2003) 1676-1683.

[22] W. Yuan and A.L. Zydney, Effects of solution environment on humic acid fouling during microfiltration, Desalination, 122 (1999) 63-76.

[23] W. Yuan and A.L. Zydney, Humic acid fouling during ultrafiltration. Environ Sci, Tech., 34 (2000) 5043-5050.

[24] W. Yuan and A.L. Zydney, Humic acid fouling during microfiltration, J. Memb. Sci., 157 (1999) 1-12.

[25] S. Sridhar, K.K. Prasad, G.S. Murthy, A.G. Rao and A.A. Khan, Processing of composite industrial effluent by reverse osmosis, J. Chem. Tech. Biotech., 78 (2003) 1061-1067.

[26] J. Schaep, B.V. der Bruggen, S. Uytterhoeven, R. Croux, C. Vandecasteele, D. Wilms, E.V. Houtte and F. Vanleberghe, Removal of hardness from ground water by nanofiltration, Desalination, 119 (1998) 295-302.

[27] R. Kettunen and P. Keskitalo, Combination of membrane technology and lime stone filtration to control drinking water quality, Desalination, 131 (2000) 271-283.

[28] W. Chen, P. Westerhoff, J.A. Leenheer and K. Booksh, Fluorescence excitation - emission matrix regional integration to quantify spectra for dissolved organic matter, Environ. Sci. Tech., 37 (2003) 5701-5710. 